\title{
Giant Cell Tumour of the Distal Ulna: A Rare Presentation
}

\author{
Ruben Jaya Kumar, MD, Vijayachandran V, MS Orth, Dinesh Ernest, MBBS, \\ Thirumal Manickam, FRCS (Edin) \\ Department of Orthopaedic, Tengku Ampuan Rahimah Hospital, Klang, Malaysia
}

\begin{abstract}
Giant-cell tumour (GCT) of bone, a primary yet locally aggressive benign tumour, commonly affects patients between the ages of 20 and 40 years, with the peak incidence occurring in the third decade. Women are affected slightly more than men. The distal end of the ulna is an extremely uncommon site for primary bone tumours in general and giant cell tumours in particular. Wide resection of the distal ulna is the recommended treatment for GCT in such locations. Radio-ulna convergence and dorsal displacement of the ulna stump are known complications following ulna resection proximal to the insertion of the pronator quadratus. This leads to reduction in grip power and forearm rotatory motion. Stabilization of the ulna stump with extensor carpi ulnaris (ECU) tendon after wide resection of the tumour has been described in the literature. We report a case of GCT of distal end of ulna treated with wide resection and stabilization with ECU tendon.
\end{abstract}

Key Words:

Giant Cell Tumour, En-bloc Resection, Distal Ilna, Extensor Carpi Ulnaris Stabilisation

\section{INTRODUCTION}

Giant-cell tumour (GCT) of the bone is a rare, benign but locally aggressive tumour, accounting for $3 \%$ to $5 \%$ of all primary bone tumours with less than $3 \%$ located at the distal ulna ${ }^{1}$. Based on tumour grading, treatment typically ranges from curettage alone to curettage with adjuvant therapy, wide resection or amputation ${ }^{2}$. The goals of treatment are adequate removal of the tumour to lower the risk of recurrence and achievement of good functional outcome. We report here the case of a patient with GCT of the distal end of the ulna, treated with en-bloc resection of the distal ulna followed by stabilization of the ulna stump using the extensor carpi ulnaris (ECU) tendon.

\section{CASE REPORT}

A 32-year-old right-handed Bangladeshi male construction worker, presented with painful progressive swelling over the ulna aspect of his right distal forearm over the past year. There was no history of any other swelling and no constitutional symptoms. There was a diffuse oval swelling of the distal right forearm along the ulnar aspect measuring $8 \times 6 \mathrm{~cm}$. The mass was firm in consistency and tender with normal overlying skin and temperature. The range of motion of the right wrist was normal except for forearm supination, which was slightly reduced. Grasp power was equal in both hand and distal neurovascular status was normal.

Haematological and serum biochemistry studies were within normal limits. Plain radiograph of the right wrist showed an expansile multiloculated lytic lesion at the distal end of the ulna without any periosteal reaction (Figure 1a). No evidence of lung metastases was seen on the chest radiograph. MRI showed heterogeneous signal intensity ranging from low to intermediate in both $\mathrm{T} 1 \mathrm{~W}$ and $\mathrm{T} 2 \mathrm{~W}$ images with adjacent infiltrated subcutaneous tissue (Figure $1 b)$.

Based on clinical and radiological findings, a working diagnosis of GCT of the distal ulna was made which was confirmed by operative biopsy results. The condition and treatment modalities were discussed at length with the patient. As per Enneking Staging System for benign bone tumours, the lesion was graded as Stage 3. Thus, an en-bloc resection was planned for him. About $3 \mathrm{~cm}$ margin of normal bone proximal to the tumour was resected which resulted in removal of a distal ulnar segment measuring about $10 \mathrm{~cm}$ in length. The entire pronator quadratus that was enveloping the tumour was detached from the radius and excised (Figure 2). Considering the patient's age, high functional needs and the high level of resection, he ulnar stump was stabilized using the extensor carpi ulnaris tenodesis technique described by Kayios et al (Figure 3).

The patient was in an above elbow cast in supination for two weeks before physiotherapy was instituted. The cast was then replaced with a wrist brace for one month. He regained normal function of his right hand without ulnar instability and resumed his occupation ten weeks postoperatively. After one year, right wrist range of motion was $0-70^{\circ}$ in dorsiflexion and $0-70^{\circ}$ in volar flexion. Right forearm supination was $0-60^{\circ}$ and pronation was $0-70^{\circ}$. 


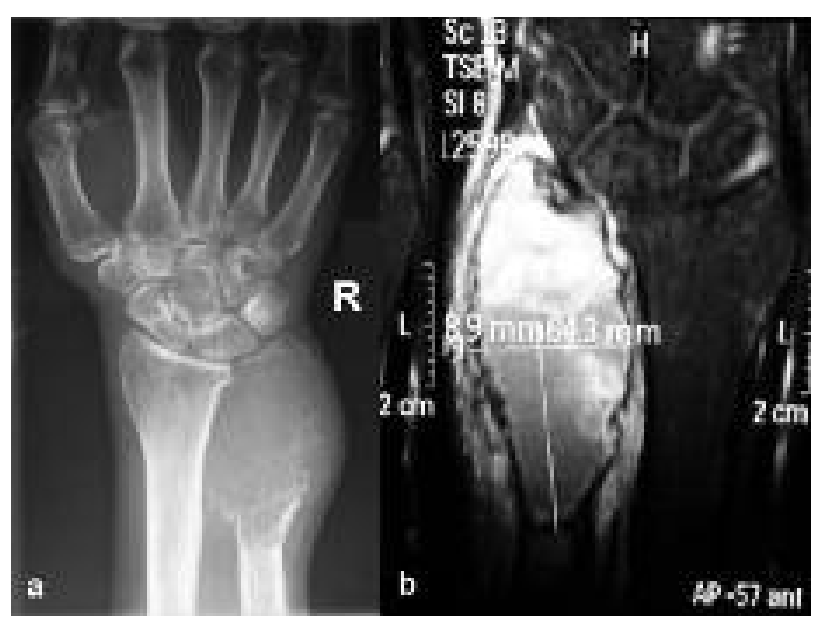

Fig. 1: (a) AP radiograph of right wrist showing expansile lytic lesion of the distal ulna. (b) MRI of the wrist. Coronal view.
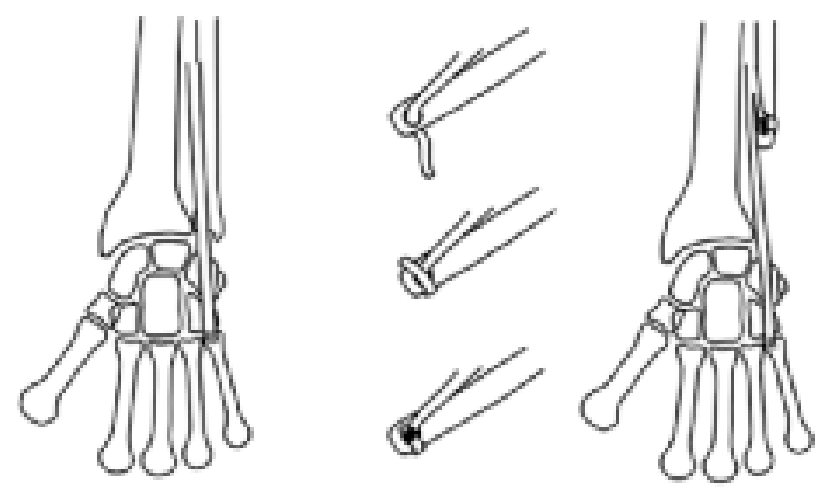

Fig. 3: Tenodesis technique using one half of ECU tendon. Reproduced with permission ${ }^{5}$.

\section{DISCUSSION}

The incidence of GCT of bone is higher in Asian populations compared to the $\mathrm{West}^{2}$. In Malaysia, $\mathrm{Ng}$ et al reported 34 (24\%) out of 141 cases of primary bone tumours were GCT and $1(2.9 \%)$ out of the 34 GCT cases involved the end of the distal ulna ${ }^{3}$. These are generally locally aggressive tumours but few become malignant with pulmonary metastases ${ }^{2}$.

The distal ulna end aids in forearm rotation and maintains the relationship between the carpus and the distal radius. However many still consider the distal region of the ulna as

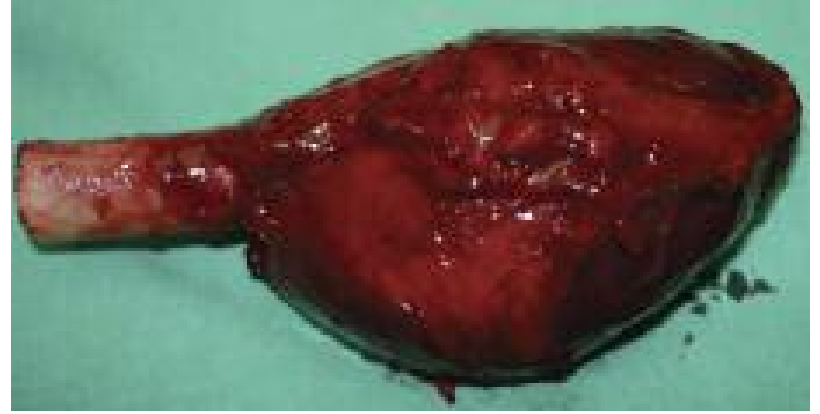

Fig. 2: Resected distal end ulna.

dispensable and its removal using the classic Darrach approach has been advocated in a variety of posttraumatic and degenerative conditions ${ }^{1}$. Cooney et al. reported good functional outcome after distal ulna resection without any stabilization and concluded that reconstruction is not routinely indicated ${ }^{4}$. On the contrary, Kayias and colleagues found a high failure rate without stabilization of the ulna stump $^{5}$, due to dorsal translation of the stump at the resection site which tends to converge towards the radius. This inevitably leads to limitation of forearm rotation, persistent pain due to instability and impingement on the radius. Thus, soft tissue stabilization of the distal ulna stump should be performed whenever possible ${ }^{5}$. Extensor carpi ulnaris (ECU) tenodesis techniques were described by Goldner et al. in 1979 , but its usage in wide resection of the distal ulna segment was first reported by Kayias and colleagues in 2006. In this study, we chose to stabilise the ulna stump after considering the length of ulna resection and removal of the entire pronator quadratus, which in our opinion could affect ulnar stability and therefore functional outcomes in this young patient.

GCT of distal ulna is a rare entity with no standard modality of treatment. We report here a case in which a patient with high functional demand had successful tumour resection complemented by ECU stabilisation. We would like to suggest that this is a viable treatment option for GCT of distal region of the ulna. 


\section{REFERENCES}

1. Singh M, Sharma S, Peshin C, Wani IH, Tikoo A, Gupta SK, et al. Wide resection and stabilisation of ulnar stump by extensor carpi ulnaris for giant cell tumour of distal ulna: two case reports. Cases $J$ 2009; 2: 8617.

2. Lim YW, Tan MH. Treatment of benign giant cell tumours of bone in Singapore. Ann Acad Med Singapore 2005; 34(3): $235-7$.

3. Ng ES, Saw A, Sengupta S. Giant cell tumour of bone with late presentation: review of treatment and outcome. J Orthop Surg 2002; 10(2): 120-8.

4. Cooney WP, Damron TA, Sim FH, Linscheid RL. En bloc resection of tumours of the distal end of the ulna. J Bone Joint Surg Am 1997; 79: 406-12.

5. Kayias EH, Drosos GI. Resection of the distal ulna for tumours and stabilization of the stump. Acta Orthop Belg 2006; 72(4): 484-91. 